\title{
Mitochondrial uncouplers inhibit hepatic stellate cell activation
}

\author{
Eduardo L Guimarães ${ }^{1}$, Jan Best ${ }^{1}$, Laurent Dollé ${ }^{1}$, Mustapha Najimi ${ }^{2}$ Etienne Sokal ${ }^{2}$ and Leo A van Grunsven ${ }^{1 *}$
}

\begin{abstract}
Background: Mitochondrial dysfunction participates in the progression of several pathologies. Although there is increasing evidence for a mitochondrial role in liver disease, little is known about its contribution to hepatic stellate cell (HSC) activation. In this study we investigated the role of mitochondrial activity through mild uncoupling during in vitro activation of HSCS.

Methods: Cultured primary human and mouse HSCs were treated with the chemical uncouplers FCCP and Valinomycin. ATP levels were measured by luciferase assay and production of reactive oxygen species was determined using the fluorescent probe DCFH-DA. Possible cytotoxicity by uncoupler treatment was evaluated by caspase 3/7 activity and cytoplasmic protease leakage. Activation of HSCs and their response to the pro-fibrogenic cytokine TGF- $\beta$ was evaluated by gene expression of activation markers and signal mediators using RT-qPCR. Proliferation was measured by incorporation of EdU and protein expression of a-smooth muscle actin was analyzed by immunocytochemistry and western blot.
\end{abstract}

Results: FCCP and Valinomycin treatment mildly decreased ATP and reactive oxygen species levels. Both uncouplers increased the expression of mitochondrial genes such as Tfam and COXIV while inducing morphological features of quiescent mouse HSCs and abrogating TGF- $\beta$ signal transduction. Mild uncoupling reduced HSC proliferation and expression of pro-fibrogenic markers of mouse and human HSCS.

Conclusions: Mild mitochondrial uncoupling inhibits culture-induced HSC activation and their response to profibrogenic cytokines like TGF- $\beta$. These results therefore suggest mitochondrial uncoupling of HSCs as a strategy to reduce progression of liver fibrosis.

Keywords: Hepatic stellate cell, Mitochondria, Uncoupler, Fibrosis

\section{Background}

Hepatic stellate cells (HSCs) are liver pericytes, located between hepatocytes and sinusoidal endothelial cells, participating in different aspects of liver physiology, with a fundamental function in vitamin A metabolism [1]. HSCs are extensively studied due to its role as the major extracellular matrix producing cell upon hepatic injury, playing a key role in the progression of chronic liver diseases. In the healthy liver it presents a so-called quiescent phenotype, containing lipid droplets rich in retinol and with a balanced extracellular matrix production. Upon chronic liver injury HSCs activate, a process where the cell loses its quiescent phenotype and acquires an

\footnotetext{
* Correspondence: Ivgrunsv@vub.ac.be

'Department of Cell Biology, Liver Cell Biology Lab, Vrije Universiteit Brussel, Laarbeeklaan 103, Brussels 1090, Belgium

Full list of author information is available at the end of the article
}

activated myofibroblastic one. This phenotype presents a high proliferation rate, produces large amounts of extracellular matrix and inflammatory cytokines, stimulating a positive feedback of inflammatory cell recruitment and collagen deposition [2].

Mitochondria are well established contributors to alcohol induced liver disease. For instance, chronic alcohol intake reduces ATP levels in hepatocytes through decreased translation of mitochondrial proteins or damaging mitochondrial DNA, further increasing cell damage $[3,4]$. Recently, mitochondria activity has also been recognized as a possible factor in the development of non-alcoholic fatty liver disease (NAFLD) [5]. One of the first links between NAFLD and mitochondria disturbance came from studies showing that obesity induces the expression of uncoupling proteins in 
hepatocytes, which decrease the mitochondrial proton gradient and hepatic ATP levels [6]. Reduced ATP levels can sensitize and induce hepatocyte apoptosis and aggravate liver injury [5].

Even though mitochondrial uncoupling and ATP depletion is associated with different pathologies, several studies indicate that mild uncoupling, a modest decrease in ATP levels, can elicit a number of physiological beneficial effects when applied to diseases related to obesity, such as diabetes and NAFLD. For example, chemical uncouplers, molecules that stimulate the leakage of protons through the mitochondrial inner membrane, are known to induce mitochondrial biogenesis, a process that ameliorates diabetes type 2 [7]. Mild mitochondrial uncoupling also inhibits lipid accumulation in adipocytes, indicating such mechanism as an appealing anti-obesity strategy [8]. Interestingly, mild chemical uncoupling can mimic the effects of calorie restriction, increasing life span and reducing reactive oxygen species (ROS) levels in mice [9]. Although the consequences of mitochondrial activity have been extensively studied in hepatocytes, little is known about its role in HSC physiology. We studied the effect of mild mitochondrial uncoupling in HSCs using the two different chemical uncouplers Carbonyl cyanide-p-trifluoromethoxyphenylhydrazone (FCCP) and Valinomycin. We show that mild chemical uncoupling is able to reduce several aspects of HSC activation indicating that HSC mitochondrial metabolism may be a new target in the prevention of liver fibrosis.

\section{Methods}

\section{Isolation of mouse and human hepatic stellate cells}

Animals were used in accordance with institutional ethical guidelines. The mouse HSC isolation method, from approximately 20 week old male mice (30 grams), used in this study was previously described [10]. Human HSCs were isolated as follows: human liver nonparenchymal cells were obtained from the left liver segment originating from a healthy cadaveric donor. These cells were obtained after two step collagenase perfusion of the liver segment, filtration and two low speed centrifugations of the cell suspension [11]. Next, human HSCs were isolated by nycodenz (Myegaard, Oslo, Norway) gradient centrifugation. For protein analyzes, $250 \times 10^{3}$ human HSCs were plated in $10 \mathrm{~cm}^{2}$ dishes, while $40 \times 10^{3}$ and $20 \times 10^{3}$ were plated in 6 well and 24 well plates for RNA and proliferation analyzes, respectively. The protocol and experiments were approved by the ethical committees of the St-Luc Hospital and faculty of Medicine of Université Catholique de Louvain.

After isolation, human and mouse cells were cultured in Dulbecco's modified Eagle's medium with 10\% fetal bovine serum at $37^{\circ} \mathrm{C}$, in a humidified atmosphere with $5 \% \mathrm{CO}_{2}$. Treatment with chemical uncouplers or DMSO as a vehicle was performed from day one of culture and after every two days, until cells were collected for the different assays. Bright field images were taken with an Axioskop light microscope (Carl Zeiss, Zaventem, Belgium).

\section{RNA expression}

After treatment with uncouplers and transforming growth factor- $\beta$ (TGF- $\beta$ ) (R\&D Systems), total RNA was extracted using the RNeasy Mini-kit (Qiagen, Hilden, Germany). RNA was reverse-transcribed using the RevertAid $^{\mathrm{m}}$ Premium Reverse Transcriptase (Fermentas, St. Leon-Rot, Germany), performed at $25^{\circ} \mathrm{C}$ for $10 \mathrm{~min}-$ utes and at $37^{\circ} \mathrm{C}$ for 40 minutes. For human HSCs, Maxima ${ }^{\circledR}$ SYBR green qPCR Master Mix (Fermentas) was used. For the semi-quantitative PCR of mouse HSCs we used an Applied Biosystems 7500 Real-Time PCR System (Applied Biosystems, Foster City, CA, USA). Primers (view Table 1 for details) were produced by Invitrogen (Paisley, UK) and probes by Roche (universal probe library, Mannheim, Germany). A 2x Maxima Probe qPCR Master Mix was used (Fermentas, St. Leon-Rot, Germany) to analyze gene expression as described previously [12].

\section{Western blotting}

Cells were exposed to lysis buffer $(170 \mathrm{mM} \mathrm{NaCl}, 10 \mathrm{mM}$ EDTA, $50 \mathrm{mM}$ Tris pH 7.4, $50 \mathrm{mM} \mathrm{NaF}, 0.2 \mathrm{mM}$ dithiothreitol and $0.5 \%$ NP-40) plus protease and phosphatase inhibitors. Protein concentration was measured using a bicinchoninic acid (BCA) determination kit (Pierce Chemical Co., Rockford, IL, USA). Ten microgram of protein was separated on $12 \%$ Tris-glycine SDS-Polyacrylamide gels and transferred onto polyvinyldifluoride (PVDF) membranes (Amersham Biosciences, Little Chalfont, UK) using a semidry blotting apparatus (Apollo ${ }^{\mathrm{TM}}$, Continental Lab Products, San Diego, CA, USA). Following, membranes were blocked in 5\% milk in TBS-Tween. After overnight incubation with primary antibodies at $4^{\circ} \mathrm{C}$ (anti$\alpha$-smooth muscle actin, 1/1000; anti- $\beta$-actin, $1 / 10000$, both from Sigma, St Louis, MO, USA) and 1 hour incubation with horseradish peroxidase conjugated secondary antibodies (1/20000) (Dako, Glostrup, Denmark), proteins were visualized with the ECL chemiluminescence detection system (Pierce Chemical Co.). Densitometry analysis was performed using Image $\mathrm{J}$.

\section{Immunocytochemistry}

Freshly isolated mouse and human HSCs were cultivated on glass cover slips in a 24 well plate and fixed with $4 \%$ paraformaldehyde for 10 minutes. Cells were permeabilized by $0.1 \%$ Triton-X 100 (in PBS with $1 \%$ bovine serum albumin) for 30 minutes followed by washing. Mouse HSCs were incubated with monoclonal antibody against $\alpha$-Smooth Muscle actin ( $\alpha$-SMA) coupled with 
Table 1 Primer sequences, probes and accession numbers of transcripts, used for RT PCR quantification

\begin{tabular}{|c|c|c|c|c|}
\hline Gene & Primers sequence (Left primer/Right primer) & Probe & GenBank accession number & Product lenght \\
\hline \multirow[t]{2}{*}{$18 \mathrm{~S}$} & 5'-aaatcagttatggttcctttggtc-3'/ & 55 & AY_248756 & 67 \\
\hline & 5'gctctagaattaccacagttatccaa-3' & & & \\
\hline \multirow[t]{2}{*}{ PGC1a } & 5'- cagtcgcaacatgctcaag-3'/ & 6 & NM_008904 & 73 \\
\hline & $5^{\prime}-$ tggggtcatttggtgactct-3' & & & \\
\hline \multirow[t]{2}{*}{ Tfam } & 5'- caaaggatgattcggctcag-3'/ & 97 & NM_009360 & 92 \\
\hline & $5^{\prime}-$ aagctgaatatatgcctgcttttc-3' & & & \\
\hline \multirow[t]{2}{*}{ CoxIV } & 5'- tcactgcgctcgttctgat-3'/ & 7 & NM_37829.1 & 67 \\
\hline & 5'- cgatcgaaagtatgagggatg-3' & & & \\
\hline \multirow[t]{2}{*}{ Ndufs7 } & 5'- gtggtgaccaagctggatg-3'/ & 104 & NM_029272 & 67 \\
\hline & 5'- cgaaggtcataggccacag-3' & & & \\
\hline \multirow[t]{2}{*}{$\mathrm{HO}-1$} & 5'- gtcaagcacagggtgacaga-3'/ & 4 & NM_010442 & 77 \\
\hline & 5'- atcacctgcagctcctcaaa-3' & & & \\
\hline \multirow[t]{2}{*}{ Smad6 } & $5^{\prime}-$ gttgcaacccctaccacttc - $3^{\prime} /$ & 70 & NM_008542 & 76 \\
\hline & 5'- ggaggagacagccgagaata -3' & & & \\
\hline \multirow[t]{2}{*}{ Smad7 } & 5'- acccccatcaccttagtcg -3'/ & 63 & NM_001042660 & 75 \\
\hline & $5^{\prime}-$ gaaaatccattgggtatctgga $-3^{\prime}$ & & & \\
\hline \multirow[t]{2}{*}{ a-SMA } & 5'-ccagcaccatgaagatcaag-3'/ & 58 & NM_007392 & 70 \\
\hline & 5'-tggaaggtagacagcgaagc-3' & & & \\
\hline \multirow[t]{2}{*}{ Pdgfr $\beta$} & 5'-tgcagagacctcaaaaggtg-3'/ & 63 & NM_008809.1 & 112 \\
\hline & 5'- cctgatcttcctcccagaaa-3' & & & \\
\hline \multirow[t]{2}{*}{ Procol1a1 } & 5'-acctaagggtaccgctgga-3'/ & 19 & NM_007742 & 97 \\
\hline & $5^{\prime}$-tccagcttctccatctttgc-3' & & & \\
\hline \multirow[t]{2}{*}{ a-SMA* } & $5^{\prime}$-ctgttccagccatccttcat-3'/ & & NM_001141945 & 70 \\
\hline & 5'-tcatgatgctgttgtaggtgg-3' & & & \\
\hline \multirow[t]{2}{*}{ PROCOL1A1* } & 5'-gacacagaggtttcagtgg-3'/ & & NM_000088.3 & 264 \\
\hline & 5'-cacccttagcaccaacag-3' & & & \\
\hline \multirow[t]{2}{*}{$185^{*}$} & 5'-aagacggaccagagcgaaag-3'/ & & K03432 & 98 \\
\hline & 5'-tcggaactacgacggtatct-3' & & & \\
\hline \multirow[t]{2}{*}{ PDGFR- $\beta^{*}$} & 5'-cccttatcatcctcatcatgc-3'/ & & NM_002609.3 & 60 \\
\hline & 5'-ccttccatcggatctcgtaa-3' & & & \\
\hline
\end{tabular}

18518 S ribosomal RNA, PGC1 a Peroxisome proliferative activated receptor gamma, coactivator 1a, Tfam Transcription factor A, mitochondrial, CoxIV Cytochrome c oxidase subunit IV, Ndufs7 NADH dehydrogenase (ubiquinone) Fe-S protein 7, HO-1 Heme oxygenase-1, Smad6 MAD homolog 6, Smad7 MAD homolog 7, a-SMA a-smooth muscle actin, $P d g f r \beta$ Platelet-derived growth factor receptor $\beta$, Procol1a1 Procollagen 1a1. * primers for human samples.

Cy3 while human HSCs were incubated with unlabeled anti- $\alpha$-SMA (both from Sigma-Aldrich, St. Louis, MO, USA), at $4^{\circ} \mathrm{C}$, overnight. After washing with PBS, human HSCs were incubated with secondary antibody conjugated with Alexa 488 (1:300) (Molecular Probes, Eugene, USA) for 1 hour at room temperature. Following washing and mounting with ProLong ${ }^{\circledR}$ Gold antifade reagent with DAPI (Invitrogen), cells were analyzed by fluorescent microscopy (Carl Zeiss, Zaventem, Belgium).

\section{Measurement of ROS}

Intracellular ROS levels were determined by measuring the fluorescence of $2^{\prime}, 7^{\prime}$ - dichlorofluorescein diacetate
(DCFH-DA) as described previously [10]. In experiments using FCCP and Valinomycin (purchased from SigmaAldrich, St. Louis, MO, USA), cells were pre-incubated for the indicated time points with uncouplers. Cell samples were analyzed by using a fluorometer (Wallac 1420 Victor multilabel counter, Wallac Oy, Turku, Finland) with $485 \mathrm{~nm}$ excitation and $535 \mathrm{~nm}$ emission wavelenghts.

\section{Viability assay}

Cell toxicity and apoptosis induction upon mitochondrial uncoupler treatment was measured using the CytoTox-Fluor $^{\text {тм }}$ Cytotoxicity Assay and Caspase 3/7 Assay (Promega, Madison, USA) as indicated in the 
manufactures protocol. In brief, one thousand human or mouse HSCs were plated in dark 96 well plates and treated with increasing concentrations of FCCP, Valinomycin or cycloheximide (Sigma-Aldrich). After 2 and 24 hours, cells were loaded with the cytotoxicity fluorescent marker bis-alanylalanyl-phenylalanyl-rhodamine 110 and incubated at $37^{\circ} \mathrm{C}$ for 30 minutes. Following, fluorescence was quantified (Wallac 1420). Subsequently, cells were lysed and loaded with a luminogenic caspase-3/7 substrate and incubated for more 30 minutes at room temperature, followed by quantification of light emission as a function of caspase 3/7 activity.

\section{Cell proliferation assay}

Cell proliferation was analyzed by measuring DNA synthesis with the Click-it EdU Cell Proliferation Assay Kit (Invitrogen). 3,750 cells per $\mathrm{cm}^{2}$ were plated in the presence or absence of FCCP or Valinomycin in combination with Tin protoporphyrin IX dichloride (SnPP) (Tocris Bioscience, USA) from the first day of culture. After 24 hours, EdU labeling was initiated. After 18 or 48 hours, for human and mouse HSCs respectively, cells were formalin fixed and visualization of the EdU incorporation was obtained according to the manufacturer's instructions.

\section{ATP levels measurement}

ATP levels were determined using the ATP Determination Kit (Invitrogen). Briefly, 3000 cells/well were plated in white 96 well plates (Sigma, St. Louis, MO, USA). After two days in culture, cells were treated with FCCP and Valinomycin for 24 hours, followed by ATP determination as determined by manufacturer's instructions.

\section{Statistical analysis}

Statistical analyzes was performed using SPSS 16.0 (SPSS Inc., Chicago, USA). Data are expressed as mean \pm standard mean. Differences among groups were analyzed for statistical significance by one-way ANOVA followed by Tukey. Results were considered significant when $p<0.05$. All data shown are representative results of at least three independent experiments.

\section{Results}

Chemical uncoupling reduces ATP and ROS levels in HSCs while inducing genes involved in mitochondrial biogenesis

We determined whether FCCP and Valinomycin were able to reduce cellular ATP levels in freshly isolated mouse HSCs, one of the main features of chemical uncoupling. As shown in Figure 1A, 24 hours treatment with either FCCP or Valinomycin, two mitochondrial uncouplers with different modes of action, reduced 30\% of ATP levels when compared to control cells. Next, we evaluated if uncouplers can modulate ROS, as they play a major role during HSC activation stimulating several pathways related to the activation process [13]. After 30 minutes, FCCP and Valinomycin reduced ROS levels to $50 \%$ and $80 \%$ of control cells respectively (Figure 1B). Interestingly, while FCCP shows a time dependent effect, Valinomycin shows no time dependent decrease in ROS levels, inducing a continuous reduction up to 24 hours. Following, we studied the effect of HSC uncoupling on genes related to mitochondrial biogenesis. These genes are stimulated when energy demand is high and have been shown to decrease ROS production and oxidative cell damage [14]. Peroxisomal proliferator activator receptor- $\gamma$ coactivator- $1 \alpha(\mathrm{Pgc}-1 \alpha)$ is a regulator of mitochondrial biogenesis, interacting with several transcriptional factors, including mitochondrial transcription factor A (Tfam), a master regulator of mitochondrial biogenesis [15]. As shown on Figure 1C, treatment with FCCP and Valinomycin increased about 15 and 10 times the expression of Pgc-1 $\alpha$, respectively. Uncouplers also increased the expression of Tfam, NADH dehydrogenase [ubiquinone] iron-sulfur protein 7 (Ndufs7) and cytochrome c oxidase subunit IV (CoxIV), the last two being genes that encode proteins from the electron transport chain (Figure 1D). These results indicate that chemical uncoupling leads to mild reduction in ATP levels and reduction in ROS levels while stimulating transcription of mitochondrial biogenesis related genes in HSCs.

\section{Chemical uncouplers reduce the expression of pro- fibrogenic genes in HSCs}

HSC activation is characterized by a phenotypic transformation from a small, lipid droplet containing cell, to a myofibroblastic phenotype, during which the cell loses its lipid droplets and acquires a large body size. The cell morphology of primary mouse HSCs treated for 7 days with chemical uncouplers was similar to that of freshly isolated quiescent HSCs (Figure 2A). As shown in the inserts, uncoupler treated cells presented a small round body size while control cells showed the typical activated myofibroblastic phenotype after 7 days of cell culture.

HSC activation is accompanied by the increased expression of pro-fibrogenic genes that are involved in several characteristics of the myofibroblastic HSC such as cell contraction and collagen deposition (Figure 2B, left graph). Uncoupler-treated cells showed a reduction of approximately $80 \%$ in the expression of $\alpha$-SMA, a standard marker of HSC activation, and $\operatorname{Pdgfr} \beta$, a receptor involved in HSC proliferation (Figure 2B, right graphic). Next, we evaluated by immunohistochemistry if $\alpha$-SMA protein expression was reduced in mouse HSCs treated with FCCP or Valinomycin. As seen in Figure 2C, $\alpha$ SMA protein expression is down regulated by both uncoupler treatments. 

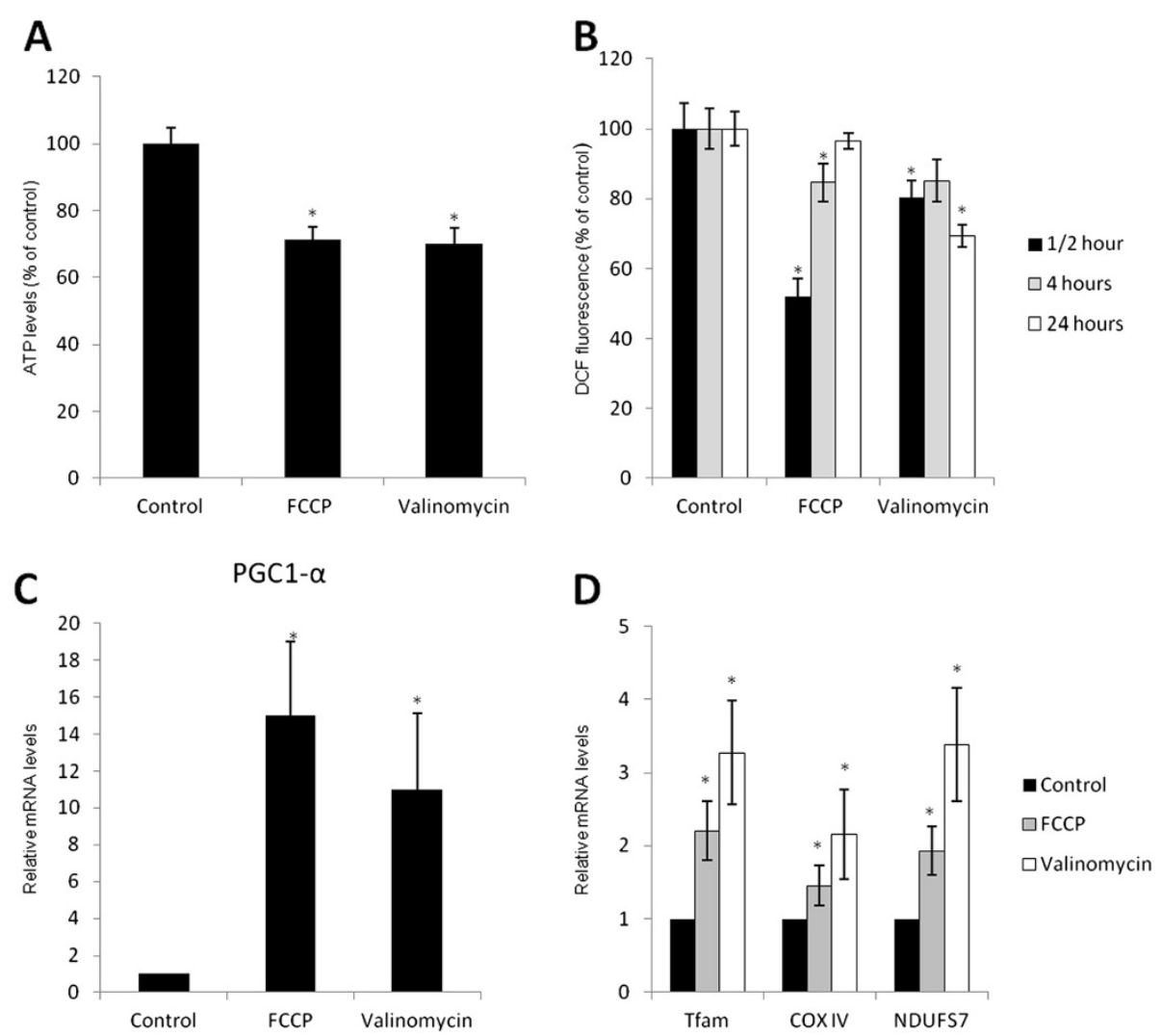

Figure 1 FCCP and Valinomycin reduce HSC ATP levels, ROS production and stimulate mitochondrial biogenesis related genes. (A) Cells were treated with $5 \mu \mathrm{M}$ of FCCP or Valinomycin for 24 hours and ATP levels were measured using a luciferase based ATP determination kit. (B) ROS levels were analyzed in $\mathrm{MHSCS}$ treated for different time points with chemical uncouplers by DCF fluorescence. HSCs were treated with $5 \mu \mathrm{M}$ of FCCP or Valinomycin for 7 days and the expression of genes involved in mitochondrial biogenesis PGC-1a (C), Tfam, Ndufs7 and CoxIV (D) were analyzed by RT-qPCR. * indicates $P<0.05$ compared to control groups. Data are expressed as means of 3 independent experiments \pm SEM.

Mild chemical uncoupling does not induce cytotoxic effects or apoptosis

In order to test whether the concentrations of uncouplers used to inhibit HSC activation could be eliciting cytotoxicity, we treated cells with a wide concentration range of FCCP and Valinomycin and analyzed a possible induction of necrosis by addition of a fluorescent substrate of cytoplasmic endopetidases. As demonstrated in Figure 3A, FCCP and Valinomycin did not elicit necrosis on any concentration tested. Actually, FCCP and Valinomycin protected against cell death on most tested concentrations. Additionally, we observed no increase in apoptosis, as measured by caspase $3 / 7$ activity, even with concentrations 10-20 fold higher than the one used to inhibit HSC activation (Figure 3B).

\section{FCCP inhibits HSC proliferation through activation of heme-oxygenase 1}

HSC proliferation is characteristic of HSC activation and a key feature in the progression of liver fibrosis. Hence, we investigated whether chemical uncoupling could reduce cell proliferation in HSCs. FCCP and Valinomycin treatment drastically decreased DNA incorporation of EdU, a BrdU analogue (Figure 4A). While control cells showed about 30\% EdU positive cells during in vitro activation, FCCP and Valinomycin treated cells showed only 8\% EdU positive cells, indicating less proliferation (Figure 4B).

Heme oxygenase $1(\mathrm{HO}-1)$ is the rate limiting enzyme in the degradation of heme groups and decreases cell proliferation when induced during HSC activation [16]. Therefore, we were interested in studying its expression during culture induced activation and its possible participation in the effect of both chemical uncouplers. HO-1 gene expression is down regulated during in vitro $\mathrm{HSC}$ activation and its expression can be rescued by FCCP and Valinomycin treatment (Figure $4 \mathrm{C}$ and $\mathrm{D}$, respectively). To test if HO-1 plays a role in the anti-proliferative effect of the uncouplers we co-treated cells with Snprotoporphyrin IX (SnPP, $5 \mu \mathrm{M})$, an inhibitor of HO-1. Co-treatment partially abrogated FCCP inhibition of proliferation (Figure 4A and 4B). Curiously, the observed Valinomycin effect was not affected by SnPP co-treatment, 


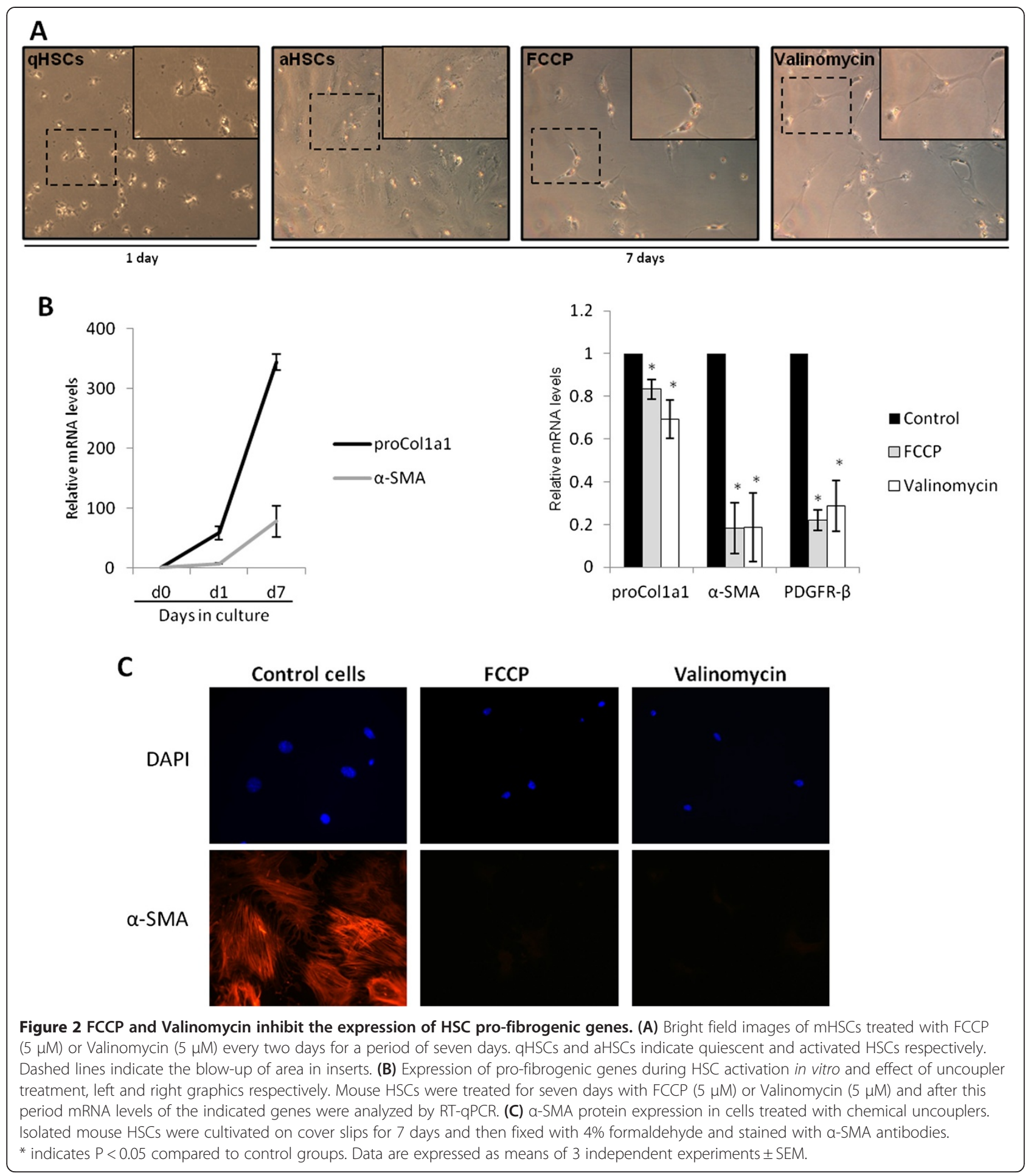

indicating that HO-1 does not play a role in the antiproliferative effect of this chemical uncoupler.

\section{Induction of pro-fibrotic genes by TGF- $\beta$ is inhibited by} chemical uncoupling

TGF- $\beta$ signalling induces several pro-fibrogenic genes in HSCs, participating in the activation process and liver fibrosis. Mitochondrial uncoupling is able to inhibit several signalling pathways in different cell types; hence we tested if mitochondrial uncoupling could affect TGF- $\beta$ signalling in HSCs. A 24 hour treatment of 2 day old HSCs with TGF- $\beta$ induced the expression of $\alpha$-SMA, Procollagen $1 \mathrm{a} 1$ and $\operatorname{Pdgfr} \beta$, while in cells co-treated with Valinomycin and FCCP 


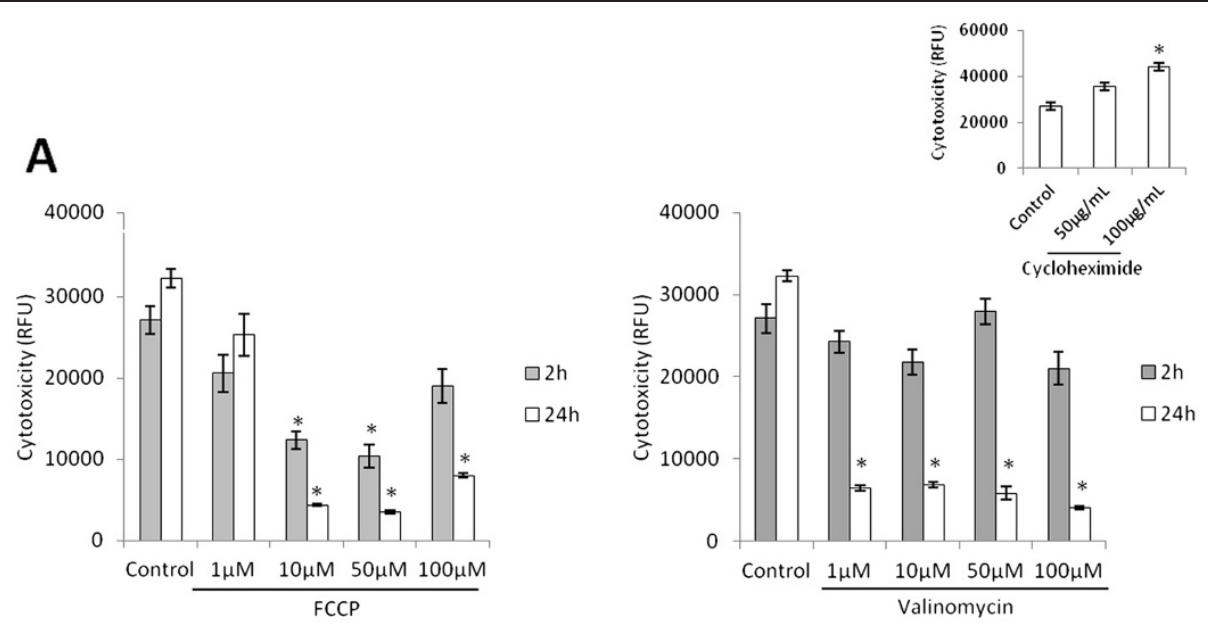

B
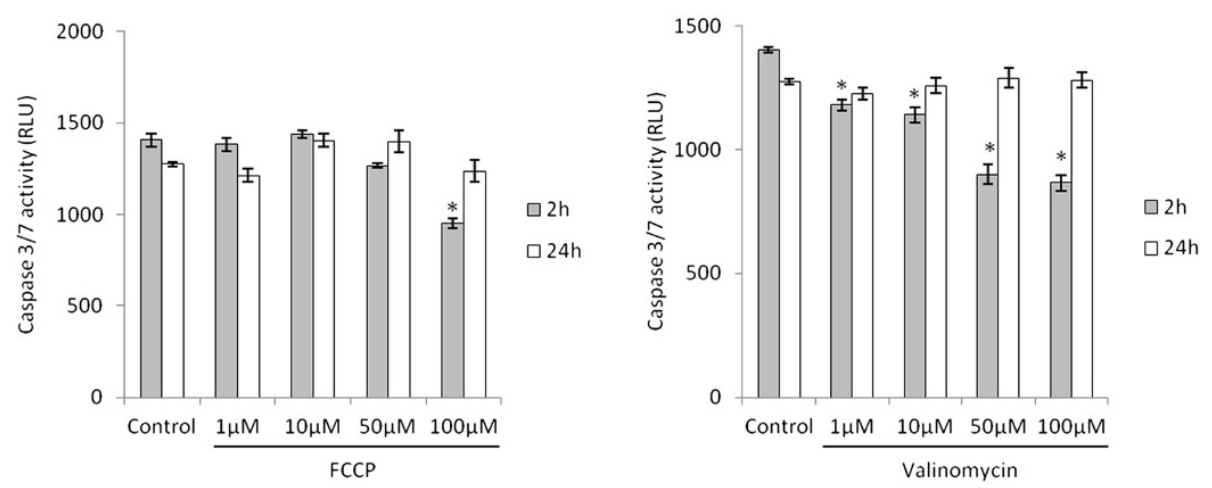

Figure 3 Chemical uncoupling does not induce cytotoxicity on concentrations that inhibit HSC in vitro activation. (A) Cytotoxic effect of mitochondrial uncouplers, as measured by fluorescence of bis-alanylalanyl-phenylalanyl-rhodamine 110, a peptide substrate of cytoplasmic peptidases. Insert shows cells treated with cycloheximide as positive control (B) Caspase 3/7 activity after treatment of cells with different concentrations of Valinomycin and FCCP on different time points. Enzyme activity was measured as described in material and methods.

* indicates $P<0.05$ compared to control group.

this TGF- $\beta$ induced effect was largely inhibited (Figure 5A).

In order to analyze whether uncouplers directly affect TGF- $\beta$ signalling, we studied if early immediate responsive genes for TGF- $\beta$, namely Smad6 and 7 , were also being inhibited. As shown in Figure 5B, while TGF- $\beta$ alone induced the expression of both genes after 2 hours of treatment, FCCP and Valinomycin inhibited such upregulation, suggesting that both uncouplers affect TGF- $\beta$ signalling.

\section{Mitochondrial uncouplers reduce activation of human HSCs}

We next evaluated if mitochondrial uncoupling was also able to reduce activation of primary human HSCs. First, we tested if human derived HSCs presented any cytotoxicity upon uncoupler treatment. High concentrations, tenfold higher than the one used to inhibit HSC activation, did not elicit increased cytotoxicity as measured by leakage of cytoplasmic peptidases and induced activation of caspase $3 / 7$ only after 24 hours treatment (Additional file 1 : Figure S1). Secondly, as shown on Figure 6A, both uncouplers induce a robust decrease in the expression of profibrogenic genes after 5 days of treatment. A marked inhibition of $\alpha$-SMA protein expression was also observed for both treatments (Figure 6B, C). As with primary mouse HSCs, we observed a decrease in the incorporation of EdU in FCCP and Valinomycin treated human HSCs (Figure 6D), indicating a decrease in cell proliferation. These results suggest that mitochondrial uncoupling cannot only reduce culture induced activation of mouse HSCs, but also reduces the fibrogenic phenotype of human activated HSCs.

\section{Discussion}

Patients with NAFLD present mitochondrial ultrastructural alterations that might reflect mitochondrial impairment and subsequent hepatocyte apoptosis [17]. 
A

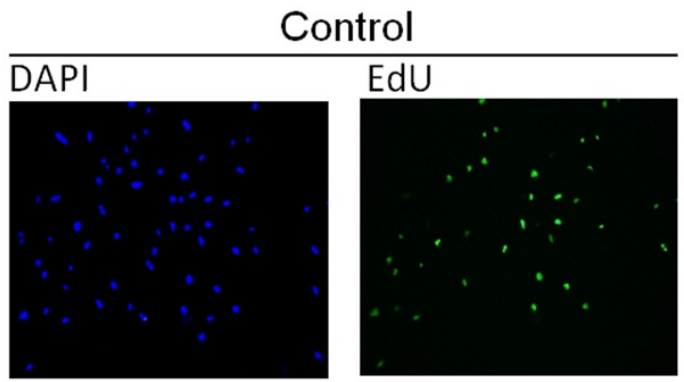

FCCP

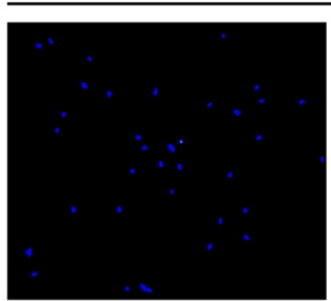

Valinomycin
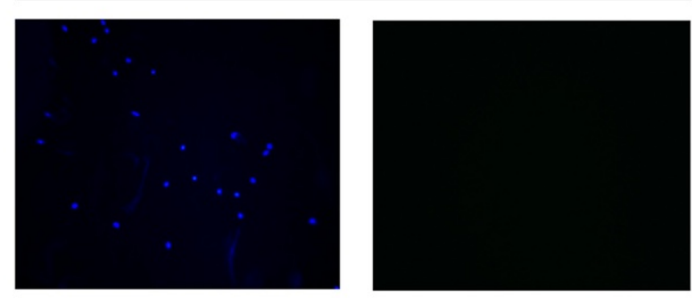

B

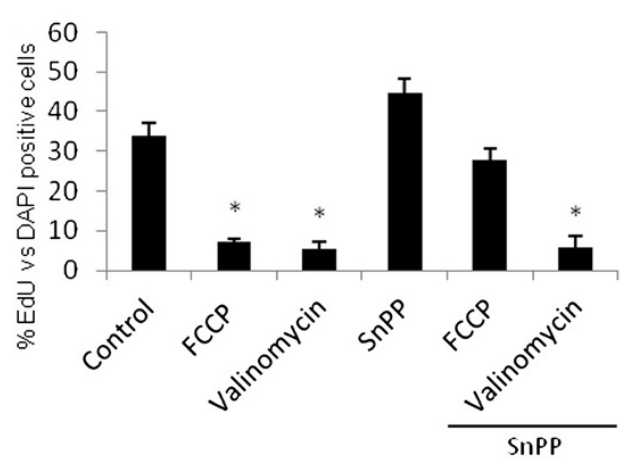

D

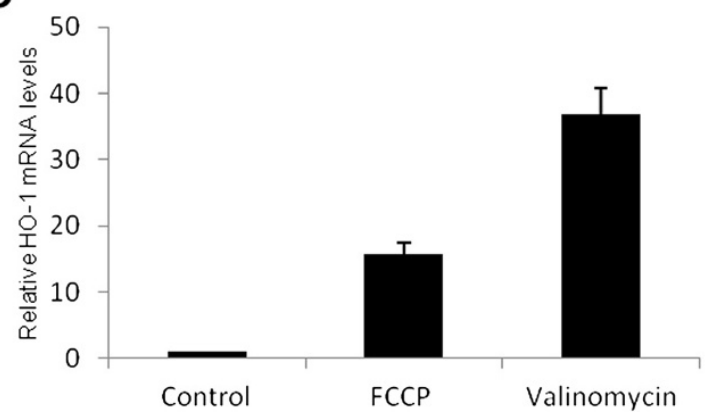

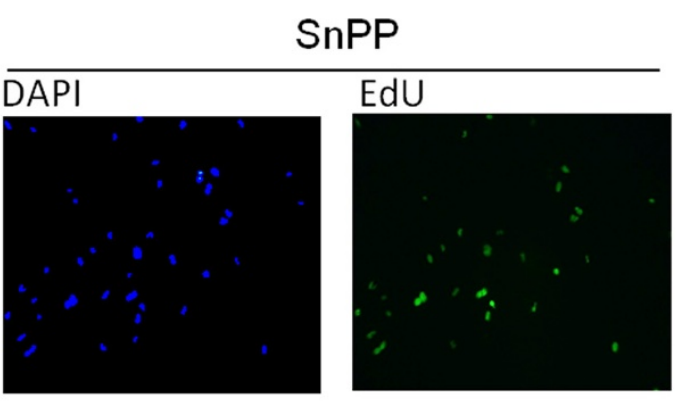

$\mathrm{FCCP}+\mathrm{SnPP}$

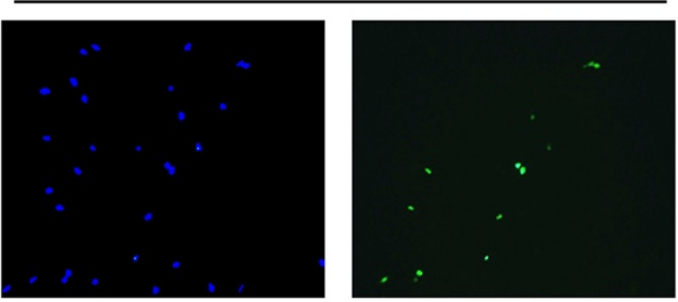

Valinomycin+SnPP
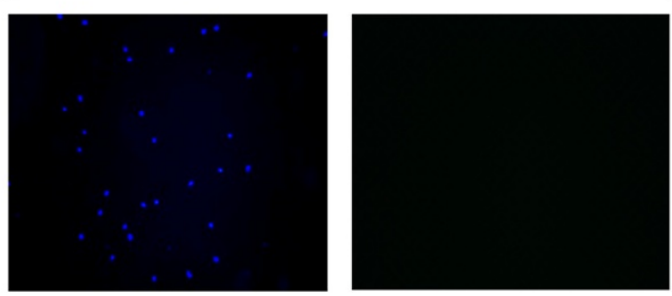

C

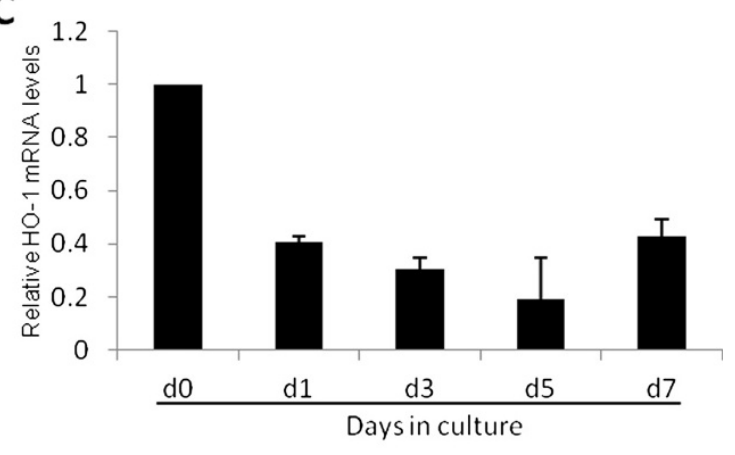

Figure 4 (See legend on next page.) 
(See figure on previous page.)

Figure 4 FCCP inhibits HSC proliferation through HO-1. (A) Representative images of cells stained with DAPI (blue fluorescence, left column) and $\mathrm{EdU}$, as a measurement of DNA synthesis (green fluorescence, right column) at day 5. Cells were treated for 5 days with uncouplers (5 $\mu \mathrm{M}$ of each) alone or in combination with the heme-oxygenase inhibitor SnPP as indicated. (B) Quantification of proliferation (EdU positive) as percentage of DAPI positive cells visualized in (A). (C) HO-1 gene expression determined by RT-qPCR during in vitro activation of primary mouse HSCs and comparing 7 day old control cells versus cells treated for 7 days with FCCP or Valinomycin (D). Data are expressed as means of 3 independent experiments \pm SEM.

Although there is mounting evidence for a mitochondrial role during liver disease, few studies have focused on the role of this organelle during HSC activation $[18,19]$. To our knowledge, this is the first demonstration that mild mitochondrial uncoupling can reduce several aspects of HSC activation.

Mitochondria can elicit cell signalling and influence cell function by adapting metabolism, altering cell response to cytokines and nuclear gene expression [20]. Although initially counter intuitive, several studies indicate that uncoupling of the mitochondrial electron transport chain can elicit beneficial cell adaptations and

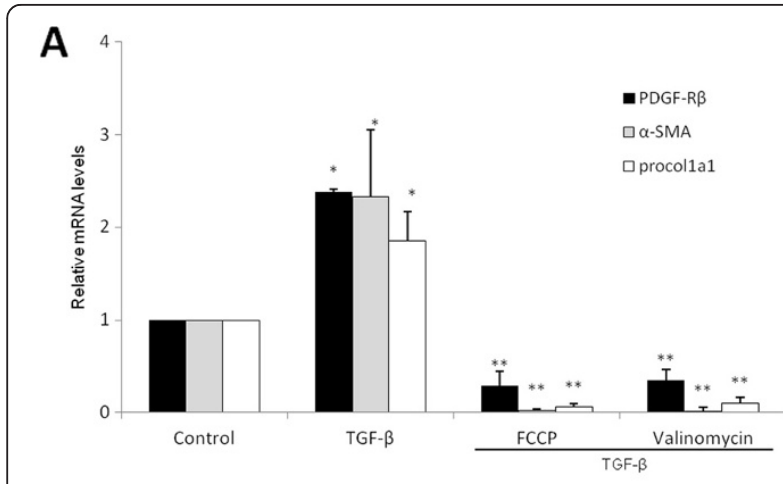

B

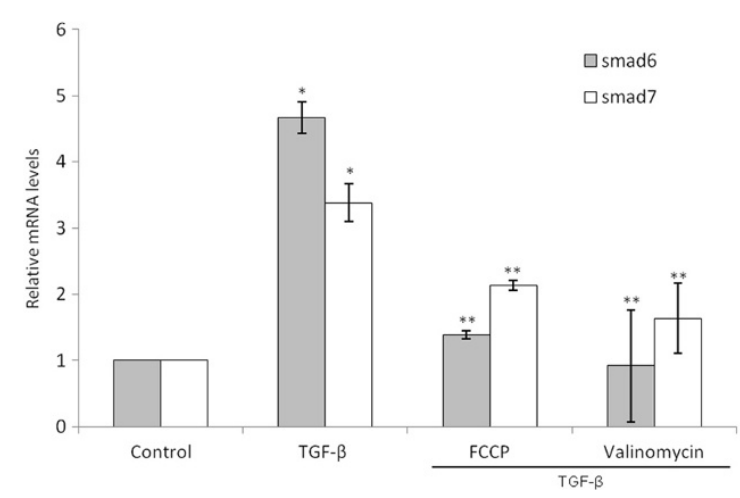

Figure 5 FCCP and Valinomycin inhibit TGF- $\beta$ signaling in 2 day old $\mathrm{mHSCs}$. (A) Expression of pro-fibrogenic genes in cells treated for 24 hours with $5 \mu \mathrm{M}$ of FCCP and Valinomycin or together with TGF- $\beta(10 \mathrm{ng} / \mathrm{mL})$ as indicated. (B) Expression of TGF- $\beta$ early immediate genes Smad6 and Smad7 on HSCs treated for 2 hours with TGF- $\beta .{ }^{*}$ indicates $P<0.05$ compared to control groups and ** indicates $P<0.05$ compared to TGF- $\beta$ group. Data are expressed as means of 3 independent experiments \pm SEM. improve several pathologies. Heart ischemia/reperfusion detrimental effects can be ameliorated through preconditioning with mild uncoupling using chemical uncouplers while studies on mice show that uncoupler treatment can increase life span, reduce oxidative stress and improve insulin levels [21]. Remarkably, a study by Rohas et al. has shown that once mild uncoupling is set, cells trigger a compensatory mechanism where PGC- $1 \alpha$ is activated and orchestrate a signalling cascade to compensate the reduction in ATP levels [22]. We also observe an increase in PGC- $1 \alpha$ expression during HSC mild uncoupling and a protective effect against cell necrosis and apoptosis in both human and mouse derived HSCs.

ROS and consequent oxidative stress participate in HSC activation through several mechanisms such as glutathione depletion and activation of transcription factors [23]. Indeed, the collagen type I promoter can be regulated by ROS generation in HSCs, demonstrating a fundamental role for oxidative stress in liver fibrosis perpetuation [24]. We observed a decrease in ROS levels in cells treated with uncouplers, suggesting ROS reduction as one possible mechanism by which these molecules inhibit HSC activation. Additionally, this result also indicates that treatment with FCCP and Valinomycin induces only mild uncoupling, since harmful uncoupling is associated with high amounts of ROS and oxidative stress $[25,26]$. Curiously, FCCP and Valinomycin show different antioxidant efficiencies. Valinomycin had a stable and less efficient effect on reducing ROS levels, while FCCP reduced ROS drastically after 30 minutes, reaching control levels after 24 hours. FCCP and Valinomycin uncouple mitochondria by different mechanisms. While FCCP is a proton mobile carrier that acidifies the mitochondrial matrix, Valinomycin acts as a specific potassium ionophore leading to alkalinisation of the mitochondrial matrix. This different effect on matrix $\mathrm{pH}$ can influence the dissimilar modulation of ROS levels [27]. Since both chemical uncouplers affect ATP levels by the same extend, it also indicates that the mode by which they affect matrix $\mathrm{pH}$ does not influence the net result on mitochondrial uncoupling.

Transcriptional regulation by the so-called adipogenic transcription factors is essential to keep HSC quiescence and has been shown to, once stimulated, inhibit several aspects of HSC activation [28]. Hence, it is hypothesized 


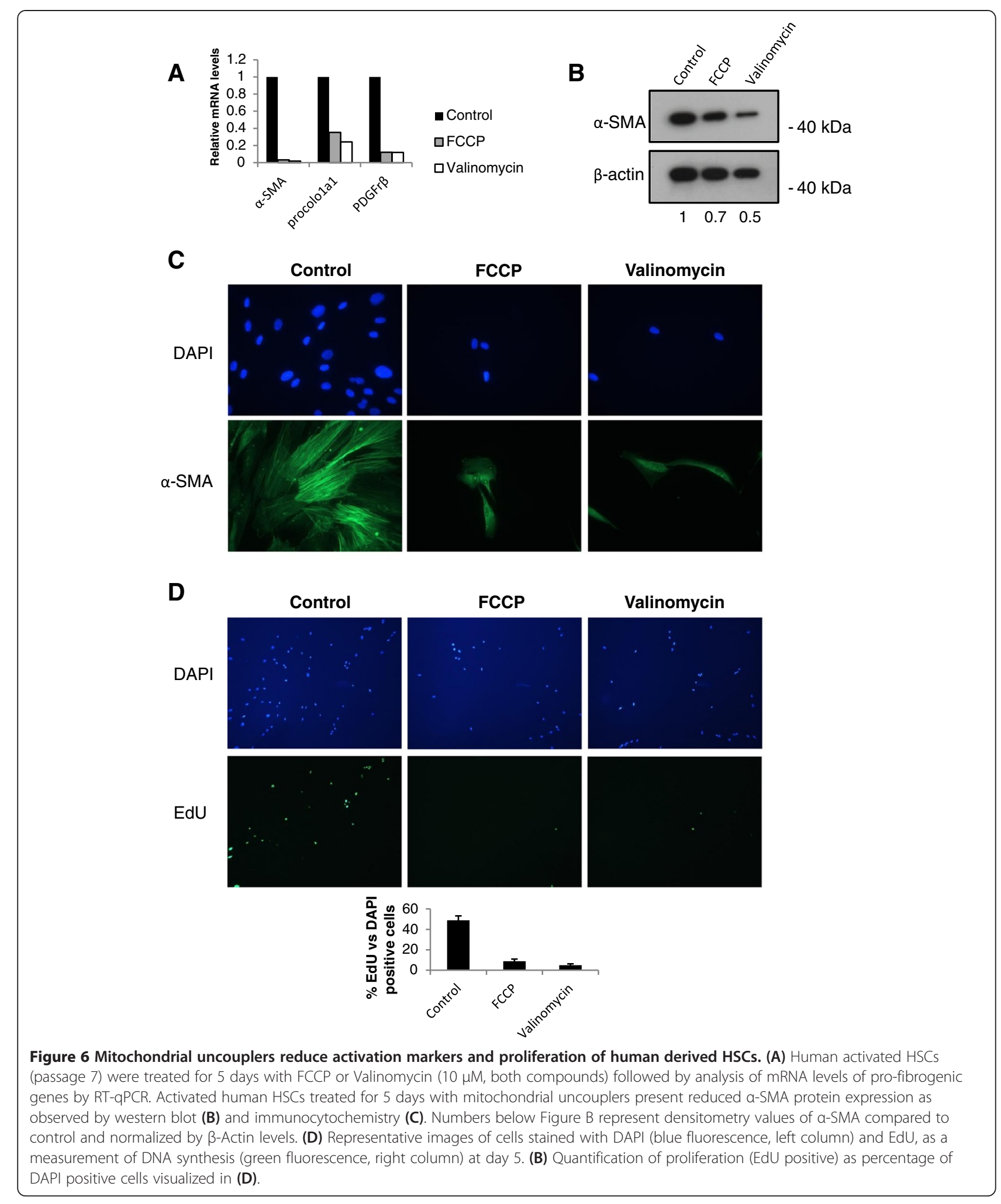

that signalling pathways leading to adipocyte differentiation similarly act on HSC activation [29]. Brown adipocytes also share analogous signalling pathways during differentiation, but opposite to white adipocytes, they present small disperse lipid droplets in the cytoplasm and activate mitochondrial uncoupling when thermogenesis is needed. We show that chemical uncoupling inhibits HSC activation and consequently stimulates the 
maintenance of the quiescent phenotype. Curiously, treatment of white adipocytes with chemical uncouplers induces the opposite of what is seen in HSCs, stimulating de-differentiation, (i.e. loss of lipid droplet and inducing a fibroblastic phenotype) [8]. Moreover, AMP-activating kinase (AMPK), a kinase with a main role in maintaining cellular energy levels, induces differentiation of brown adipocytes when chronically activated, while it inhibits the differentiation of white adipocytes [30]. In HSCs, AMPK inhibits several aspects of HSC activation and stimulates quiescent characteristics [31]. Together, these data and the results observed in this study indicate that the HSC activation process shares analogous signalling pathways with the differentiation of brown adipocytes, cells that present mitochondrial uncoupling activity and, more importantly, a lipogenic phenotype similar to the one of quiescent HSCs.

We show that different aspects of human and mouse HSC activation such as $\alpha$-SMA expression and proliferation are inhibited by chemical mitochondrial uncoupling. One important aspect of activated HSC is the capacity to proliferate and consequently aggravate chronic liver injury. HO-1, an enzyme involved in the metabolism of heme, can reduce HSC proliferation mainly due to the production of bilirubin, an antioxidant end product of heme group degradation [16]. Curiously, both uncouplers inhibit the down regulation of HO-1 seen during activation, but HO-1 is only responsible for the antiproliferative effect of FCCP and not Valinomycin, as shown using the HO-1 inhibitor SnPP. It has been reported that Valinomycin is able to inhibit the proliferation of different cell lines, although the mechanism is still not fully understood [32]. Our results show that, in general, uncoupling can inhibit HSC proliferation, but the mechanism diverges depending on the mode of mitochondrial uncoupling.

TGF- $\beta$ plays an essential role during liver fibrosis, and has been intensely investigated as a target for therapy. This cytokine activates HSC in vivo, and TGF- $\beta$ KO models have shown a dramatic reduction in $\alpha$-SMA positive cells in liver fibrosis with a consecutive decrease in collagen deposition [33]. In this study we observed an increase in the expression of several fibrogenic genes when HSCs were treated with TGF- $\beta$ which was completely abrogated by co-treatment with chemical uncouplers. The inhibition of TGF- $\beta$ induced early-immediate genes Smad6/7 by the uncouplers suggests a direct influence on TGF- $\beta$ signalling. Although it is not clear how mild mitochondrial uncoupling can influence TGF- $\beta$ signalling, it is known that this cytokine can induce the release of mitochondrial calcium stores and that this process is necessary for the activation of protein kinases and downstream signalling of TGF- $\beta$ [34]. Chemical uncoupling can influence calcium release from mitochondria
[35] and therefore might influence TGF- $\beta$ signalling through modulation of mitochondrial calcium stores.

Intriguingly, the observations in this study add a new perspective to the action of some molecules that are already known to inhibit HSC activation. For example, curcumin has been shown to be a powerful mitochondrial uncoupler in the same concentration range known to inhibit HSC fibrogenic features [36,37].

\section{Conclusion}

The present study shows, for the first time, that mild mitochondrial uncoupling can inhibit HSC activation. Importantly, we also observed a reduction in fibrogenic features of human derived HSCs. Contrarily to mouse HSCs, which were treated with FCCP and Valinomycin still at the quiescent phenotype, the human HSCs used in this study presented an activated phenotype when treated (passage 7). Nonetheless, we still observed a decrease in fibrogenic features on both gene and protein levels. These last results show that mild mitochondrial uncoupling, additionally to its capacity to inhibit the first steps of HSC activation, is able to induce a decrease in activation features of already myofibroblastic HSCs. This is an important characteristic when considering possible applications in the treatment of liver fibrosis. In summary, we show that mild mitochondrial uncoupling can inhibit several aspects of HSC activation and indicates HSC mitochondrial metabolism as a possible new target for liver fibrosis therapy.

\section{Additional file}

Additional file 1: Figure S1. Mitochondrial uncouplers show no cytotoxicity at low concentrations. (A) Cytotoxic effect of mitochondrial uncouplers on human HSCs, as measured by fluorescence of bisalanylalanyl-phenylalanyl-rhodamine 110, a peptide substrate of cytoplasmic peptidases. (B) Caspase $3 / 7$ activity after treatment of cells with different concentrations of Valinomycin and FCCP on different time points. Enzyme activity was measured as described in material and methods. ${ }^{*}$ indicates $P<0.05$ compared to control group.

\section{Competing interests}

The authors declare that there is no duality of interest associated with this manuscript.

\section{Authors' contributions}

ELG: study concept, experimental design, acquisition of data, drafting of the manuscript. JB and LD: acquisition and isolation of human HSCS. MN and ES: acquisition of human liver donors and liver material; LAvG: study supervision, experimental design and critical revision of the manuscript. All authors read and approved the final manuscript.

\section{Acknowledgements}

We would like to thank Tom Schouteet for his technical assistance. The work was funded by a Ph.D. grant from the research council of the Vrije

Universiteit Brussel to E. L.M. Guimarães, an IWT project (SBO, "Hepstem") and a European Union FP7/Cosmetics Europe cofunded project (HeMiBio, ECGA \#266777 only related to the human HSC experiments) to L.A. van Grunsven and funding from the Brussels region (ISRIB/"Brustem") to L.A. van Grunsven and E. Sokal. 


\section{Author details}

${ }^{1}$ Department of Cell Biology, Liver Cell Biology Lab, Vrije Universiteit Brussel, Laarbeeklaan 103, Brussels 1090, Belgium. '2Laboratory of Pediatric Hepatology and Cell Therapy, Université Catholique de Louvain (UCL), Brussel, Belgium.

Received: 6 January 2012 Accepted: 23 April 2012

Published: 11 June 2012

\section{References}

1. Friedman SL: Hepatic Stellate Cells: Protean, Multifunctional, and Enigmatic Cells of the Liver. Physiol Rev 2008, 88:125-172.

2. Atzori L, Poli G, Perra A: Hepatic stellate cell: A star cell in the liver. Int J Biochem Cell Biol 2009, 41:1639-1642.

3. Coleman WB, Cunningham CC: Effects of chronic ethanol consumption on the synthesis of polypeptides encoded by the hepatic mitochondrial genome. Biochim Biophys Acta - Bioenerg 1990, 1019:142-150.

4. Cahill A, Wang X, Hoek JB: Increased Oxidative Damage to Mitochondrial DNA Following Chronic Ethanol Consumption. Biochem Biophys Res Commun 1997, 235:286-290.

5. Mantena SK, King AL, Andringa KK, Eccleston HB, Bailey SM: Mitochondrial dysfunction and oxidative stress in the pathogenesis of alcohol- and obesity-induced fatty liver diseases. Free Radic Biol Med 2008, 44:1259-1272.

6. Chavin KD, Yang S, Lin HZ, Chatham J, Chacko VP, Hoek JB, Walajtys-Rode E, Rashid A, Chen C-H, Huang C-C et al: Obesity Induces Expression of Uncoupling Protein-2 in Hepatocytes and Promotes Liver ATP Depletion. J Biol Chem 1999, 274:5692-5700.

7. Fujisawa K, Nishikawa T, Kukidome D, Imoto K, Yamashiro T, Motoshima H, Matsumura T, Araki E: TZDs reduce mitochondrial ROS production and enhance mitochondrial biogenesis. Biochem Biophys Res Commun 2009, 379:43-48.

8. Tejerina S, De Pauw A, Vankoningsloo S, Houbion A, Renard P, De Longueville F, Raes M, Arnould T: Mild mitochondrial uncoupling induces 3T3-L1 adipocyte de-differentiation by a PPAR\{gamma\}-independent mechanism, whereas TNF\{alpha\}-induced de-differentiation is PPAR \{gamma\} dependent. J Cell Sci 2009, 122:145-155.

9. Camille CCdS, Fernanda MC, Lívea FB, Marisa HGM, Alicia JK: Mild mitochondrial uncoupling in mice affects energy metabolism, redox balance and longevity. Aging Cell 2008, 7:552-560.

10. Guimarães ELM, Empsen C, Geerts A, van Grunsven LA: Advanced glycation end products induce production of reactive oxygen species via the activation of NADPH oxidase in murine hepatic stellate cells. $J$ Hepatol 2010, 52:389-397.

11. Najimi M, Khuu DN, Lysy PA, Jazouli N, Abarca J, Sempoux C, Sokal EM: Adult-derived human liver mesenchymal-like cells as a potential progenitor reservoir of hepatocytes? Cell Transplant 2007, 16:717-728.

12. Mannaerts I, Nuytten NR, Rogiers V, Vanderkerken K, van Grunsven LA, Geerts A: Chronic administration of valproic acid inhibits activation of mouse hepatic stellate cells in vitro and in vivo. Hepatology 2010, 51:603-614.

13. Norifumi K, Shuichi S, Masayasu I, Tetsuo K: Effect of antioxidants, resveratrol, quercetin, and $\mathrm{N}$-acetylcysteine, on the functions of cultured rat hepatic stellate cells and kupffer cells. Hepatology 1998, 27:1265-1274.

14. Kong X, Wang R, Xue Y, Liu X, Zhang H, Chen Y, Fang F, Chang Y: Sirtuin 3, a New Target of PGC-1a, Plays an Important Role in the Suppression of ROS and Mitochondrial Biogenesis. PLoS One 2010, 5:e11707.

15. Ventura-Clapier R, Garnier A, Veksler V: Transcriptional control of mitochondrial biogenesis: the central role of PGC-1\{alpha\}. Cardiovasc Res 2008, 79:208-217.

16. Liying L, Pascale G, Jeanne Tran Van N, Boris J, Ariane M, Aamir H, Sophie L: Heme oxygenase- 1 is an antifibrogenic protein in human hepatic myofibroblasts. Gastroenterology 2003, 125:460-469.

17. Serviddio G, Sastre J, Bellanti F, Viña J, Vendemiale G, Altomare E: Mitochondrial involvement in non-alcoholic steatohepatitis. Mol Aspects Med 2007, 29:22-35.

18. Siegmund V Sr, Qian T, de Minicis S, Harvey-White J, Kunos G, Vinod KY, Hungund B, Schwabe RF: The endocannabinoid 2-arachidonoyl glycerol induces death of hepatic stellate cells via mitochondrial reactive oxygen species. FASEB J 2007, 21:2798-2806.
19. Mann J, Mann DA: Transcriptional regulation of hepatic stellate cells. Adv Drug Deliv Rev 2009, 61:497-512.

20. Butow RA, Avadhani NG: Mitochondrial Signaling: The Retrograde Response. Mol Cell 2004, 14:1-15.

21. Modrianský M, Gabrielová E: Uncouple my heart: the benefits of inefficiency. J Bioenerg Biomembr 2009, 41:133-136.

22. Rohas LM, St-Pierre J, Uldry M, Jager S, Handschin C, Spiegelman BM: A fundamental system of cellular energy homeostasis regulated by PGC-1a. Proc Natl Acad Sci 2007, 104:7933-7938.

23. Poli G: Pathogenesis of liver fibrosis: role of oxidative stress. Mol Aspects Med 2000, 21:49-98.

24. Greenwel P, Domínguez-Rosales J-A, Mavi G, Rivas-Estilla AM, Rojkind M: Hydrogen peroxide: A link between acetaldehyde-elicited a1(i) collagen gene up-regulation and oxidative stress in mouse hepatic stellate cells. Hepatology 2000, 31:109-116.

25. Han YH, Kim SW, Kim SH, Kim SZ, Park WH: 2,4-Dinitrophenol induces G1 phase arrest and apoptosis in human pulmonary adenocarcinoma Calu-6 cells. Toxicol in Vitro 2008, 22:659-670.

26. Hoehn KL, Salmon AB, Hohnen-Behrens C, Turner N, Hoy AJ, Maghzal GJ, Stocker R, Van Remmen H, Kraegen EW, Cooney GJ, et al: Insulin resistance is a cellular antioxidant defense mechanism. Proc Natl Acad Sci 2009 106:17787-17792.

27. Silic-Benussi M, Cannizzaro E, Venerando A, Cavallari I, Petronilli V, La Rocca N, Marin O, Chieco-Bianchi L, Di Lisa F, D'Agostino DM, et al: Modulation of mitochondrial $\mathrm{K}+$ permeability and reactive oxygen species production by the p13 protein of human T-cell leukemia virus type 1. Biochim Biophys Acta - Bioenerg 2009, 1787:947-954.

28. Tsukamoto $\mathrm{H}$, She $\mathrm{H}$, Hazra S, Cheng J, Wang J: Fat paradox of steatohepatitis. J Gastroenterol Hepatol 2008, 23:S104-S107.

29. Tsukamoto H, She H, Hazra S, Cheng J, Miyahara T: Anti-adipogenic regulation underlies hepatic stellate cell transdifferentiation. J Gastroenterol Hepatol 2006, 21:S102-S105.

30. Vila-Bedmar R, Lorenzo M, Fernandez-Veledo S: Adenosine 5'Monophosphate-Activated Protein Kinase-Mammalian Target of Rapamycin Cross Talk Regulates Brown Adipocyte Differentiation. Endocrinology 2010, 151:980-992.

31. Caligiuri A, Bertolani C, Guerra CT, Aleffi S, Galastri S, Trappoliere M, Vizzutti F, Gelmini S, Laffi G, Pinzani M, et al: Adenosine monophosphate-activated protein kinase modulates the activated phenotype of hepatic stellate cells. Hepatology 2008, 47:668-676.

32. Kleuser B, Rieter H, Adam G: Selective Effects by Valinomycin on Cytotoxicity and Cell Cycle Arrest of Transformed versus Nontransformed Rodent Fibroblasts in Vitro. Cancer Res 1985, 45:3022-3028.

33. Hellerbrand C, Stefanovic B, Giordano F, Burchardt ER, Brenner DA: The role of TGF[beta]1 in initiating hepatic stellate cell activation in vivo. J Hepatol 1999, 30:77-87.

34. Chow JYC, Dong H, Quach KT, Van Nguyen PN, Chen K, Carethers JM: TGF- $\beta$ mediates PTEN suppression and cell motility through calcium-dependent PKC-a activation in pancreatic cancer cells. Am J Physiol Gastrointest Liver Physiol 2008, 294:G899-G905.

35. Bernardi P: Mitochondrial Transport of Cations: Channels, Exchangers, and Permeability Transition. Physiol Rev 1999, 79:1127-1155.

36. Lim HW, Lim HY, Wong KP: Uncoupling of oxidative phosphorylation by curcumin: Implication of its cellular mechanism of action. Biochem Biophys Res Commun 2009, 389:187-192.

37. $X u J, F u ~ Y, C h e n ~ A$ : Activation of peroxisome proliferator-activated receptor-\{gamma\} contributes to the inhibitory effects of curcumin on rat hepatic stellate cell growth. Am J Physiol Gastrointest Liver Physiol 2003, 285:G20-G30.

\section{doi:10.1186/1471-230X-12-68}

Cite this article as: Guimarães et al:: Mitochondrial uncouplers inhibit hepatic stellate cell activation. BMC Gastroenterology 2012 12:68. 